SAE paper 2009-01-0578. Copyright (C) 2009 SAE International. This paper is posted on this site with permission from SAE International, and is for viewing only. Further use or distribution of this paper is not permitted without permission from SAE 


\title{
Robust Yaw Moment Control for Vehicle Handling and Stability
}

\author{
Haiping Du \\ Nong Zhang \\ Wade Smith \\ Faculty of Engineering, University of Technology, Sydney
}

Copyright $\odot 2009$ SAE International

\begin{abstract}
This paper presents a robust controller design method for improving vehicle lateral stability and handling performance. In particular, the practical load variation will be taken into account in the controller synthesis process such that the controller can keep the vehicle lateral stability and handling performance regardless of the load variation. Based on a two-degree-of-freedom (2-DOF) lateral dynamics model, a model-based TakagiSugeno fuzzy control strategy is applied to design such a controller and the sufficient conditions for designing such a controller are given in terms of linear matrix inequalities (LMIs) which can be solved efficiently using currently available numerical software. Numerical simulations are used to validate the effectiveness of the proposed control approach.
\end{abstract}

\section{INTRODUCTION}

A considerable research and subsequent developments have been done to improve vehicle handling and stability. Among them, the yaw moment control has proved its effectiveness to improve vehicle handling and stability during severe driving manoeuvres. In general, the motivation for the development of yaw moment control can be understood by examining the driver's disabilities to control vehicle directional dynamics under critical conditions. For example, in a turning manoeuvre with high lateral acceleration, where tyre forces are approaching or at the limit of road adhesion, the vehicle sideslip angle grows and the effectiveness of the vehicle steering angle in generating a yaw moment becomes significantly reduced because of the saturation of tyre force. The decrease in restoring yaw moment generated by the tyre lateral force when the sideslip angle increases may cause the unstable motion of the vehicle called the spin motion. Providing the required external yaw moment will therefore recover the stability of the vehicle.

A practical approach to generate a required external yaw moment can be the transverse distribution of the vehicle braking forces between the left and right wheels. This strategy known as differential braking can be achieved using the main parts of the common antilock braking system (ABS). Nowadays, how to design the required controller based on the measured or estimated variables to achieve the desired performance is attracting much more attention. Several authors have proposed different control methods. For example, optimal control [1,2], fuzzy logic control [3], $\mathrm{H}_{\infty}$ yaw-moment control [4], internal model control [5], multi-objective control [6,7], LQR and sliding mode control [8], etc. As the vehicle is operating under a wide range of conditions of speed, load, friction etc., the active yaw moment control should guarantee the stability performance robustly in the presence of uncertainty arising from different operating situations. In addition, the physical constraints of control input resulting from the actuation system and the tyreroad conditions, etc., may cause the saturation of the control input with possible deteriorations of the control performance. Therefore, the controller design for yaw moment control should consider both robust stability and control saturation aspects. These issues, however, are ignored or partly dealt with in those studies.

This paper concerns with the robust controller design for a two-degree-of-freedom (2DOF) vehicle lateral dynamics model considering vehicle load uncertainty

\footnotetext{
The Engineering Meetings Board has approved this paper for publication. It has successfully completed SAE's peer review process under the supervision of the session organizer. This process requires a minimum of three (3) reviews by industry experts.

All rights reserved. No part of this publication may be reproduced, stored in a retrieval system, or transmitted, in any form or by any means, electronic, mechanical, photocopying, recording, or otherwise, without the prior written permission of SAE.

ISSN 0148-7191

Positions and opinions advanced in this paper are those of the author(s) and not necessarily those of SAE. The author is solely responsible for the content of the paper.

SAE Customer Service: Tel: $\quad 877-606-7323$ (inside USA and Canada)

Tel: $\quad$ 724-776-4970 (outside USA)

Fax: $\quad 724-776-0790$

Email: CustomerService@sae.org

SAE Web Address:
} http://www.sae.org

Printed in USA 
and control input constraint. The focus of the paper is to design a state feedback control law such that the closedloop system is asymptotically stable with the optimal disturbance attenuation subject to a variation of vehicle mass and control input constraint. To deal with the vehicle load uncertainty, the vehicle lateral dynamics model is first represented by a Takagi-Sugeno (T-S) fuzzy model using "Sector Nonlinearity" approach. Then, a robust T-S fuzzy controller is designed based on the parallel distributed compensation (PDC) scheme. Sufficient conditions for designing such a controller are given in terms of linear matrix inequalities (LMIs), which can be solved very efficiently using available software like Matlab LMI Toolbox. Simulation results are used to validate the control performance.

This paper is organised as follows. In Section 2, the linear vehicle lateral dynamics model is briefly presented. In Section 3, the control problem is formulated considering the vehicle load uncertainty and control input constraint. In Section 4, the fuzzy controller development is presented. Then, in section 5 , the results of the simulation for different steering manoeuvres are shown and discussed. Finally, conclusions are presented in section 6 .

\section{LINEAR VEHICLE MODEL}

A vehicle's handling dynamics in the horizontal plane are represented by a linear two-degree of freedom (2DOF) bicycle model with states of sideslip angle $\beta$ at the centre of gravity (CG) and yaw rate $r$ as shown in Figure 1.

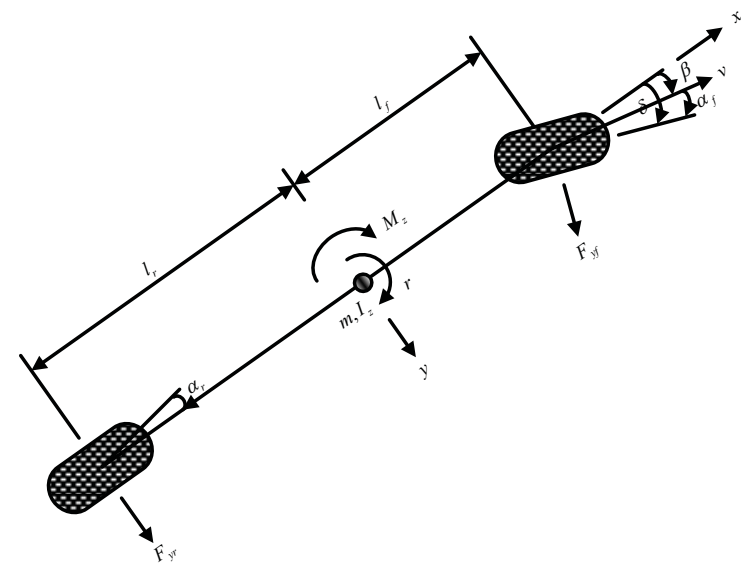

Figure 1. Bicycle model

The equations governing the lateral and yaw motions for the linear bicycle model follows from the force and moment balance can be expressed as [3]

$$
\begin{aligned}
& m v(\dot{\beta}+r)=C_{f}\left(\beta+l_{f} \frac{r}{v}-\delta\right)+C_{r}\left(\beta-l_{r} \frac{r}{v}\right), \\
& I_{z} \dot{r}=l_{f} C_{f}\left(\beta+l_{f} \frac{r}{v}-\delta\right)-l_{r} C_{r}\left(\beta-l_{r} \frac{r}{v}\right)+M_{z} .
\end{aligned}
$$

where $I_{z}$ is the moment of inertia of the vehicle about its yaw axis, $m$ is the vehicle mass, $l_{f}$ and $l_{r}$ are distance of the front and rear axles from the centre of gravity (CG). $C_{f}$ and $C_{r}$ are cornering stiffnesses of the front and rear tyres. $\delta$ is the steering angle. $M_{z}$ is yaw moment and $v$ is vehicle velocity.

Considering the vehicle velocity to be time invariant, the following state space equation can be formulated as:

$\dot{x}=A x+B_{1} w+B_{2} u$,

where $x$ is the state variable, $u$ is the control input (yaw moment $M_{z}$ ), which is generated from the difference of the brake forces between the left and right drive wheels. $w$ is the disturbance (steering angle $\delta$ ). The variables and matrices are given as

$$
\begin{aligned}
& x=\left[\begin{array}{c}
\beta \\
r
\end{array}\right], \quad A=\left[\begin{array}{cc}
-\frac{C_{f}+C_{r}}{m v} & -1-\frac{l_{f} C_{f}-l_{r} C_{r}}{m v^{2}} \\
-\frac{l_{f} C_{f}-l_{r} C_{r}}{I_{z}} & -\frac{l_{f}^{2} C_{f}+l_{r}^{2} C_{r}}{I_{z} v}
\end{array}\right], \\
& B_{1}=\left[\begin{array}{c}
\frac{C_{f}}{m v} \\
\frac{l_{f} C_{f}}{I_{z}}
\end{array}\right], B_{2}=\left[\begin{array}{c}
0 \\
\frac{1}{I_{z}}
\end{array}\right] . w=\delta, u=M_{z} .
\end{aligned}
$$

\section{PROBLEM FORMULATION}

LOAD VARIATION - In practice, the vehicle mass can be varied in terms of the loading conditions such as the number of riding persons and payload, etc. Since the uncertain vehicle mass $m$ is bounded by its minimum value $m_{\min }$ and its maximum value $m_{\max }$ in practice, using the idea of "sector nonlinearity" [9], it can thus be represented by

$$
\frac{1}{m}=h_{1}(\xi) m_{s \max }+h_{2}(\xi) m_{s \min },
$$

where $\xi=\frac{1}{m}$ is defined as a premise variable (i.e., the variable $x$ used in the if-part of a single fuzzy if-then rule 
like "if $\mathrm{x}$ is $\mathrm{A}$ then $\mathrm{y}$ is $\mathrm{B}$ "), $m_{s \max }=\frac{1}{m_{\min }}, m_{s \min }=\frac{1}{m_{\max }}$,

$h_{1}(\xi)$ and $h_{1}(\xi)$ are membership functions which are defined as

$$
h_{1}(\xi)=\frac{\frac{1}{m}-m_{s \min }}{m_{s \max }-m_{s \min }}, h_{2}(\xi)=\frac{m_{s \max }-\frac{1}{m}}{m_{s \max }-m_{s \min }} .
$$

We name the above mentioned two membership functions as "Light" and "Heavy", respectively, the uncertain vehicle handling model (2) can then be represented by the following fuzzy models.

\section{Model Rule 1:}

IF $\xi$ is light,

THEN $\dot{x}=A_{1} x+B_{11} w+B_{2} u$.

Model Rule 2:

IF $\xi$ is heavy,

THEN $\dot{x}=A_{2} x+B_{12} w+B_{2} u$.

And then, the Takagi-Sugeno (T-S) fuzzy model which exactly represents the uncertain vehicle handling model (2) under the assumption on bounds of vehicle mass $m \in\left[m_{\min }, m_{\max }\right]$ is obtained as:

$\dot{x}=\sum_{i=1}^{2} h_{i}(\xi)\left[A_{i} x+B_{1 i} w\right]+B_{2} u$,

where $h_{i}(\xi) \geq 0, i=1,2$, and $\sum_{i=1}^{2} h_{i}(\xi)=1$.

In practice, the vehicle mass $m$ can be measured, thus, the T-S fuzzy model (5) can be realised.

INPUT CONSTRAINT - On the other hand, the control law designed should be found in a way that some physical constraints of control input resulting from both the actuation system and the tyre-road conditions are satisfied. That is the calculated yaw moment must remain below the maximum admissible value determined by the maximum difference in the longitudinal forces that can be generated by the left and right wheels during every cornering manoeuvre. This indicates that the control saturation may exist in some emergency conditions and should be accounted for in the controller design. Then, equation (5) is expressed as $\dot{x}=\sum_{i=1}^{2} h_{i}(\xi)\left[A_{i} x+B_{1 i} w\right]+B_{2} \bar{u}$,

where $\bar{u}$ is the bounded input expressed as $\bar{u}=\operatorname{sat}(u)$, where $\operatorname{sat}(u)$ is a saturation function of control input $u$ and is defined as

$\operatorname{sat}(u)=\left\{\begin{array}{cc}-u_{\lim }, & \text { if } u<-u_{\lim }, \\ u & , \quad \text { if }-u_{\lim } \leq u \leq u_{\lim }, \\ u_{\lim }, & \text { if } u>u_{\lim },\end{array}\right.$

where $u_{\text {lim }}$ is a control input limit.

In order to avoid the large number of inequalities problem involved in the controller design, the norm bounded approach is used to handle the saturation nonlinearity defined in (7). Hence, the equation (6) will be written as:

$$
\begin{aligned}
\dot{x} & =\sum_{i=1}^{2} h_{i}(\xi)\left[A_{i} x+B_{1 i} w\right]+B_{2} \bar{u} \\
& =\sum_{i=1}^{2} h_{i}(\xi)\left[A_{i} x+B_{1 i} w\right]+B_{2} \frac{1+\varepsilon}{2} u+B_{2}\left(\bar{u}-\frac{1+\varepsilon}{2} u\right) \\
& =A_{h} x+B_{1 h} w+B_{2} \frac{1+\varepsilon}{2} u+B_{2} \bar{v},
\end{aligned}
$$

where

$$
A_{h}=\sum_{i=1}^{2} h_{i}(\xi) A_{i}, B_{1 h}=\sum_{i=1}^{2} h_{i}(\xi) B_{1 i}, \bar{v}=\bar{u}-\frac{1+\varepsilon}{2} u \text {. }
$$

And, we have the following lemma.

Lemma 1 For the saturation constraint defined by (7), as long as $|u| \leq \frac{u_{\text {lim }}}{\varepsilon}$, we have

$\left\|\bar{u}-\frac{1+\varepsilon}{2} u\right\| \leq \frac{1-\varepsilon}{2}\|u\|$,

and hence

$\left[\bar{u}-\frac{1+\varepsilon}{2} u\right]^{T}\left[\bar{u}-\frac{1+\varepsilon}{2} u\right] \leq\left(\frac{1-\varepsilon}{2}\right)^{2} u^{T} u$

where $0<\varepsilon<1$.

\section{CONTROLLER DESIGN}

The robust fuzzy controller design for the uncertain handling model (8) is carried out based on the so-called 
PDC scheme [9]. For the T-S fuzzy model (8), we construct the fuzzy state feedback controller via the PDC as:

$u=\sum_{i=1}^{2} h(\xi) K_{i} x=K_{h} x$

where $K_{h}=\sum_{i=1}^{2} h(\xi) K_{i}, K_{i}$ is the state feedback gain matrix to be designed.

The purpose of the yaw moment control is to bring the vehicle yaw rate response and/or the vehicle slip angle into conformance with the desired yaw rate and/or slip angle. Therefore, to improve the handling and stability of the vehicle, the yaw rate and the sideslip angle of the vehicle will be controlled to follow their target values. Generally, the desired sideslip angle is regarded as zero, i.e., $\beta_{d}=0$; the desired yaw rate has been calculated as

$r_{d}=\frac{v}{l\left(1+k_{u} v^{2}\right)} \delta$

where $l=l_{f}+l_{r}$ and $k_{u}$ is the stability factor. To make the sideslip angle and yaw rate closing to their desired values, the control output is defined as

$$
\begin{aligned}
z & =\left[\begin{array}{c}
\beta-\beta_{d} \\
r-r_{d}
\end{array}\right]=\left[\begin{array}{ll}
1 & 0 \\
0 & 1
\end{array}\right]\left[\begin{array}{l}
\beta \\
r
\end{array}\right]+\left[\begin{array}{c}
0 \\
-\frac{v}{l\left(1+k_{u} v^{2}\right)}
\end{array}\right] \delta \\
& =C_{z} x+D_{z} w
\end{aligned}
$$

And, in order to design a controller to perform adequately in a wide range of manoeuvres, the $L_{2}$ gain of the system (11) with (12) and (14), which is defined as

$$
\left\|T_{z w}\right\|_{\infty}=\sup _{\|w\|_{2} \neq 0} \frac{\|z\|_{2}}{\|w\|_{2}}
$$

where $\|z\|_{2}^{2}=\int_{0}^{\infty} z^{T} z d t$ and $\|w\|_{2}^{2}=\int_{0}^{\infty} w^{T} w d t$ and the supermum is taken over all non-zero trajectories of the system with $x(0)=0$, is chosen as the performance measure. The goal is to design a robust controller (9) such that the uncertain system (8) with controller (9) is quadratically stable and the $L_{2}$ gain (12) is minimised.

Lemma 2 For any matrices (or vectors) $X$ and $Y$ with appropriate dimensions, we have

$X^{T} Y+Y^{T} X \leq \kappa X^{T} X+\kappa^{-1} Y^{T} Y$ where $\kappa>0$ is any scalar.

To derive the conditions for designing the controller (9), we now define a Lyapunov function for the system (8) with (9) as

$$
V(x(t))=x^{T}(t) P x(t),
$$

where $P$ is a positive definite matrix. By differentiating (13), we obtain

$$
\begin{aligned}
& V(x)=\dot{x}^{T} P x+x^{T} P \dot{x} \\
& =\left[A_{h} x+B_{1} w+B_{2} \frac{1+\varepsilon}{2} u+B_{2} \bar{v}\right]^{T} P x \\
& +x^{T} P\left[A_{h} x+B_{1} w+B_{2} \frac{1+\varepsilon}{2} u+B_{2} \bar{v}\right] .
\end{aligned}
$$

By Lemma 1, Lemma 2, and definition (9), we have

$$
\begin{aligned}
& \dot{V}(x) \leq \\
& x^{T}\left[A_{h}^{T} P+P A_{h}+\left(B_{2} \frac{1+\varepsilon}{2} K_{h}\right)^{T} P+P B_{2} \frac{1+\varepsilon}{2} K_{h}\right] x \\
& +w^{T} B_{1}^{T} P x+x^{T} P B_{1} w+\kappa \bar{v}^{T} \bar{v}+\kappa^{-1} x^{T} P B_{2} B_{2}^{T} P x \\
& \leq x^{T} \Theta x+w^{T} B_{1}^{T} P x+x^{T} P B_{1} w,
\end{aligned}
$$

where

$$
\begin{aligned}
\Theta= & A_{h}^{T} P+P A_{h}+\left(B_{2} \frac{1+\varepsilon}{2} K_{h}\right)^{T} P+P B_{2} \frac{1+\varepsilon}{2} K_{h} \\
& +\kappa\left(\frac{1-\varepsilon}{2}\right)^{2} K_{h}^{T} K_{h}+\kappa^{-1} P B_{2} B_{2}^{T} P,
\end{aligned}
$$

and $\kappa$ is any positive scalar.

Adding $z^{T} z-\gamma^{2} w^{T} w$ to both sides of (14) yields

$$
\begin{aligned}
& V(x)+z^{T} z-\gamma^{2} w^{T} w \\
& \leq\left[\begin{array}{lr}
x^{T} & w^{T}
\end{array}\right]\left[\begin{array}{cc}
\Theta+C_{z}^{T} C_{z} & P B_{1}+D_{z}^{T} D_{z} \\
* & -\gamma^{2}
\end{array}\right]\left[\begin{array}{l}
x \\
w
\end{array}\right] .
\end{aligned}
$$

Let us consider

$$
\Pi=\left[\begin{array}{cc}
\Theta+C_{z}^{T} C_{z} & P B_{1}+D_{z}^{T} D_{z} \\
* & -\gamma^{2}
\end{array}\right]<0,
$$


then, $V(x)+z^{T} z-\gamma^{2} w^{T} w \leq 0$ and the $L_{2}$ gain defined in (12) is less than $\gamma>0$ with the initial condition $x(0)=0$. When the disturbance is zero, i.e., $w=0$, it can be inferred from (15) that if $\Pi<0$, then $V(x)<0$, and the fuzzy system (8) with the controller (9) is quadratically stable.

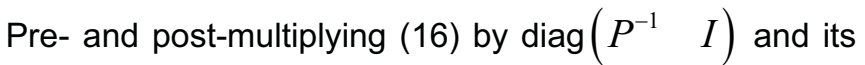
transpose, respectively, and defining $Q=P^{-1}$, $Y_{h}=K_{h} Q$, the condition $\Pi<0$ is equivalent to

$\Sigma=\left[\begin{array}{cc}Q A_{h}^{T}+A_{h} Q+\frac{1+\varepsilon}{2} Y_{h}^{T} B_{2}^{T} & \\ +\frac{1+\varepsilon}{2} B_{2} Y_{h}+\kappa\left(\frac{1-\varepsilon}{2}\right)^{2} Y_{h}^{T} Y_{h} & B_{1}+Q D_{z}^{T} D_{z} \\ +\kappa^{-1} B_{2} B_{2}^{T}+Q C_{z}^{T} C_{z} Q & \\ * & -\gamma^{2} I+D_{z}^{T} D_{z}\end{array}\right]<0$.

By the Schur complement, $\Sigma<0$ is equivalent to

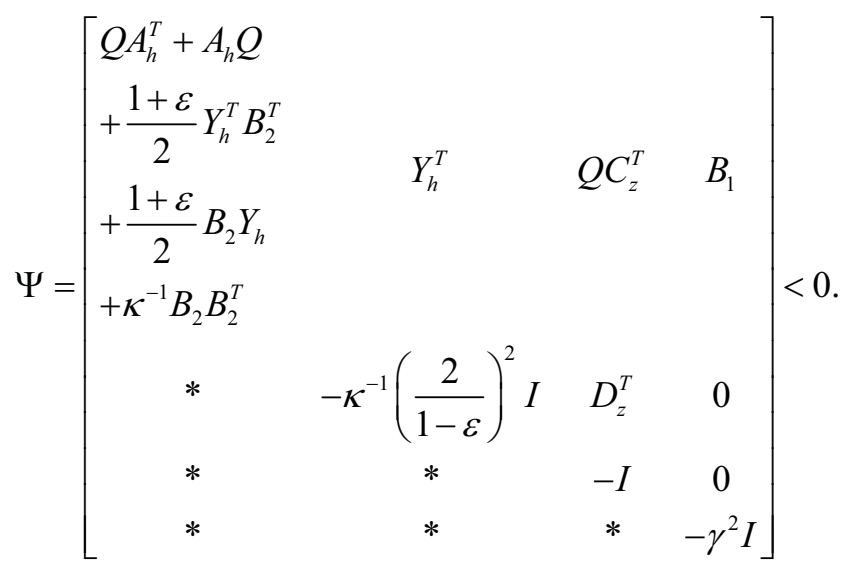

By the definitions $A_{h}=\sum_{i=1}^{2} h_{i}(\xi) A_{i}, B_{1 h}=\sum_{i=1}^{2} h_{i}(\xi) B_{1 i}$

and the fact that $h_{i}(\xi) \geq 0 \sum_{i=1}^{2} h_{i}(\xi)=1, \quad \Psi<0$ is equivalent to
$\left[\begin{array}{cccc}Q A_{i}^{T}+A_{i} Q & & & \\ +\frac{1+\varepsilon}{2} Y_{i}^{T} B_{2}^{T} & & & \\ +\frac{1+\varepsilon}{2} B_{2} Y_{i} & Y_{i}^{T} & Q C_{z}^{T} & B_{1} \\ +\kappa^{-1} B_{2} B_{2}^{T} & & & \\ * & -\kappa^{-1}\left(\frac{2}{1-\varepsilon}\right)^{2} I & D_{z}^{T} & 0 \\ * & * & -I & 0 \\ * & * & * & -\gamma^{2} I\end{array}\right]<0$.

On the other hand, from (9), the constraint $\left|u \leq \frac{u_{\mathrm{lim}}}{\varepsilon}\right|$

can be expressed as

$\left|\sum_{i=1}^{2} h_{i}(\xi) K_{i} x\right| \leq \frac{u_{\lim }}{\varepsilon}$

It is obvious that if $\left|K_{i} x\right| \leq \frac{u_{\text {lim }}}{\varepsilon}$, then (18) holds. Let $\Omega(K)=\left\{x(t)|| x^{T} K_{i}^{T} K_{i} x \mid \leq\left(\frac{u_{\text {lim }}}{\varepsilon}\right)^{2}\right\}$, the equivalent condition for an ellipsoid $\Omega(P, \rho)=\left\{x \mid x^{T} P x \leq \rho\right\}$ being a subset of $\Omega(K)$, i.e., $\Omega(P, \rho) \subset \Omega(K)$ is:

$K_{i}\left(\frac{P}{\rho}\right)^{-1} K_{i}^{T} \leq\left(\frac{u_{\mathrm{lim}}}{\varepsilon}\right)^{2}$.

By the Schur complement, inequality (19) can be written as

$\left[\begin{array}{cc}\left(\frac{u_{\mathrm{lim}}}{\varepsilon}\right)^{2} & K_{i}\left(\frac{P}{\rho}\right)^{-1} \\ * & \left(\frac{P}{\rho}\right)^{-1}\end{array}\right] \geq 0$.

Using the definitions $Q=P^{-1}$ and $Y_{i}=K_{i} Q$, inequality (20) is equivalent to

$\left[\begin{array}{cc}\left(\frac{u_{\lim }}{\varepsilon}\right)^{2} & Y_{i} \\ * & \rho^{-1} Q\end{array}\right] \geq 0$.

The minimisation of $\gamma$ can be realised as 
$\min \gamma$ subject to (17) and (21).

This problem can be solved very efficiently by means of the Matlab LMI Toolbox software.

\section{SIMULATION RESULTS}

Numerical simulation is conducted to show the effectiveness of the proposed controller. Simulation results are carried out using the linear 2DOF vehicle model and the simulation software based on MATLAB and SIMULINK. The parameters characterising the vehicle model are listed in Table 1. These parameters correspond to a typical vehicle model.

\begin{tabular}{c|l|l}
\hline Name & Explanation & Value \\
\hline$m$ & Vehicle total mass & $1298.9 \mathrm{~kg}$ \\
\hline$l_{f}$ & $\begin{array}{l}\text { Distance of CG from the } \\
\text { front axle }\end{array}$ & $1 \mathrm{~m}$ \\
\hline$l_{r}$ & $\begin{array}{l}\text { Distance of CG from the rear } \\
\text { axle }\end{array}$ & $1.454 \mathrm{~m}$ \\
\hline$h$ & $\begin{array}{l}\text { Height of the sprung mass } \\
\text { CG }\end{array}$ & $0.533 \mathrm{~m}$ \\
\hline$C_{f}$ & Front tyre cornering stiffness & $30000 \mathrm{~N} / \mathrm{rad}$ \\
\hline$C_{r}$ & Rear tyre cornering stiffness & $30000 \mathrm{~N} / \mathrm{rad}$ \\
\hline$k_{u}$ & Stability factor & 0.005 \\
\hline$I_{z}$ & $\begin{array}{l}\text { Moment of inertia about the } \\
\text { yaw axis }\end{array}$ & $1627 \mathrm{kgm}$ \\
\hline & &
\end{tabular}

Table 1: Vehicle Parameters

In the study, we suppose the output yaw moment is limited to $3000 \mathrm{Nm}$. The vehicle mass is assumed to have $20 \%$ variation of its nominal value. Using the controller design approach presented in the last section, we can obtain the controller gain matrices.

The effectiveness of the designed controller is validated considering two different steering angle inputs, one is for lane change manoeuvre as shown in Figure 2 and the other is for J-turn manoeuvre as shown in Figure 3. And to clarify the effects of the proposed controller, both the vehicle dynamics with and without controller are checked.

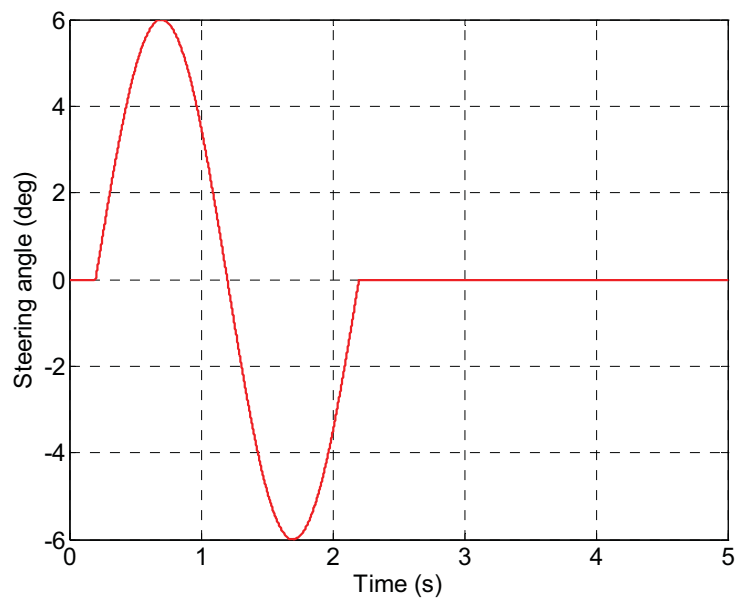

Figure 2: Steering input for a lane change manoeuvre

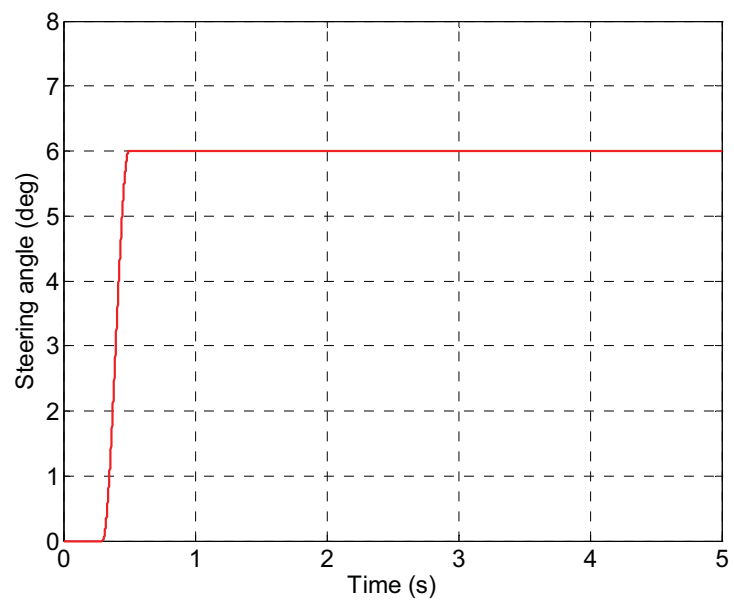

Figure 3: Steering input for a J-turn manoeuvre

Figures 4-6 show the simulation results for a lane change manoeuvre at a velocity of $30 \mathrm{~m} / \mathrm{s}$ with different vehicle mass, where Figure 4 shows the time response of sideslip angle for a controlled and uncontrolled vehicle model with different vehicle mass at a velocity of $30 \mathrm{~m} / \mathrm{s}$ and Figure 5 shows the time response of yaw rate for a controlled and uncontrolled vehicle model with different vehicle mass at a velocity of $30 \mathrm{~m} / \mathrm{s}$. To track the references, the controller generates a yaw moment $M_{z}$ shown in Figure 6 which can be generated by applying appropriate braking forces to the front and rear wheels.

From Figures 4-5, we observe that the response of the controlled system is better than the uncontrolled system regardless of the change of vehicle mass. In particularly, the sideslip angle almost exactly tracks to the reference value because we put a big weighting value on sideslip angle in the control output (11). 


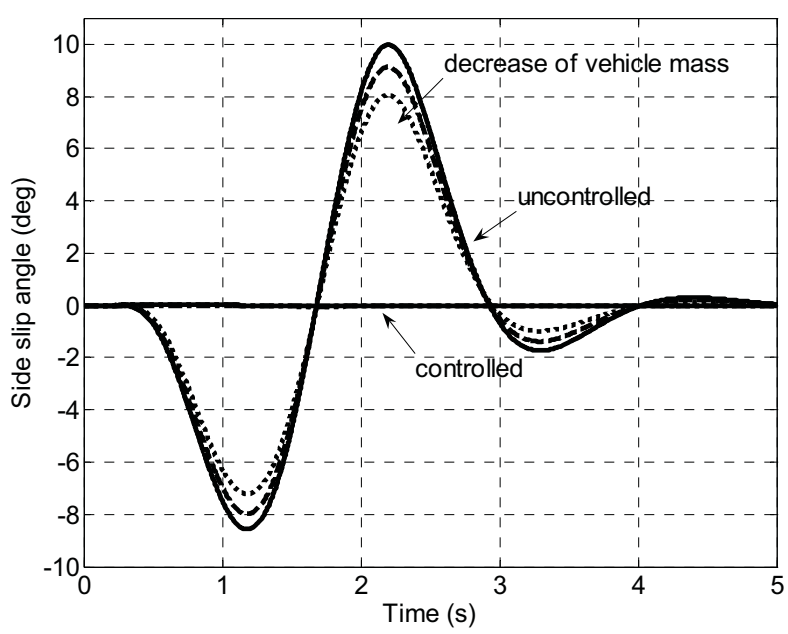

Figure 4: Simulation results for sideslip angle under lane change manoeuvre

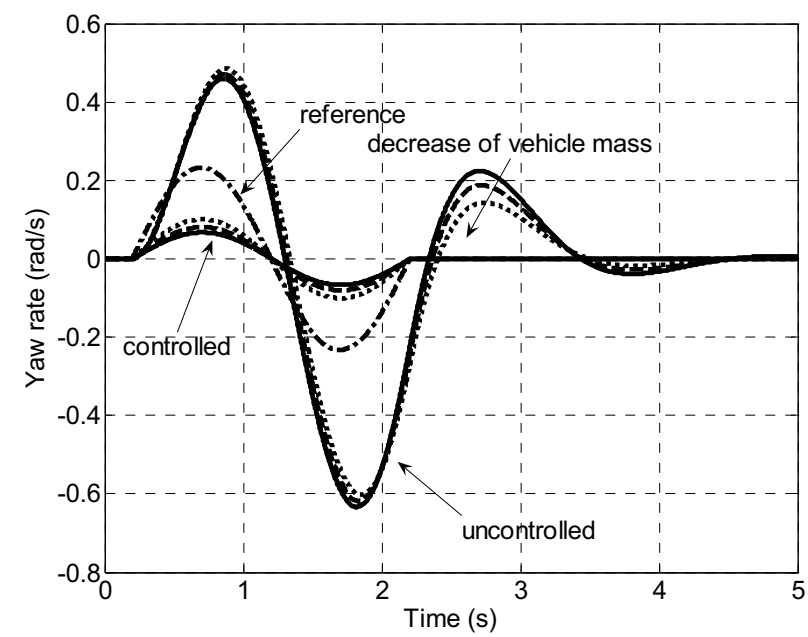

Figure 5: Simulation results for yaw rate under lane change manoeuvre

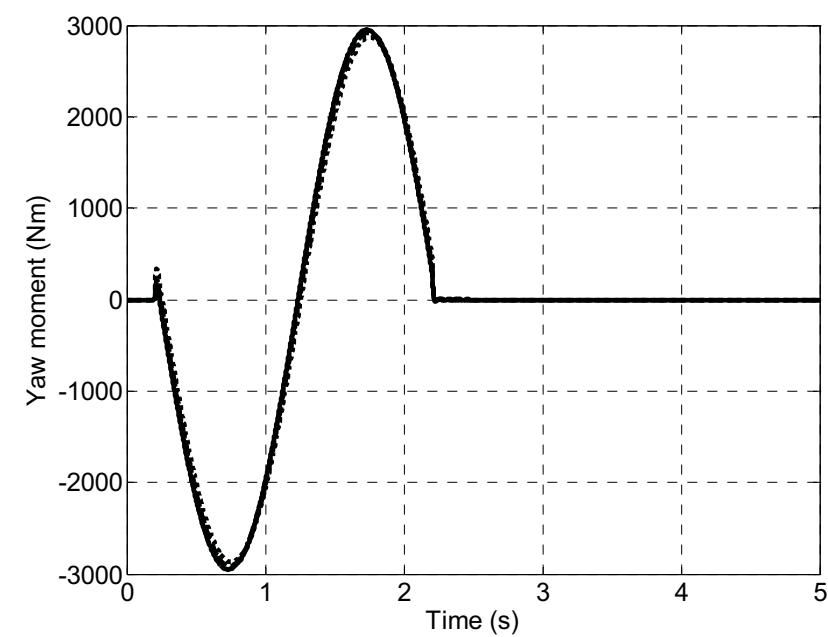

Figure 6: Simulation results for yaw moment under lane change manoeuvre
Figures 7-9 show other simulations for a J-turn manoeuvre at a velocity of $30 \mathrm{~m} / \mathrm{s}$. In the simulations, the J-turn manoeuvre is produced from the ramp steer input ( $\delta=6 \mathrm{deg}$ ) as shown in Figure 3 .

In the simulations, the responses of sideslip angle and yaw rate for the controlled vehicle show better performance than the uncontrolled vehicle in spite of the change of vehicle mass as shown in the previous simulations. As demonstrated by the simulation results, the proposed robust controller enables stability control in the presence of vehicle mass uncertainty and actuator output constraint.

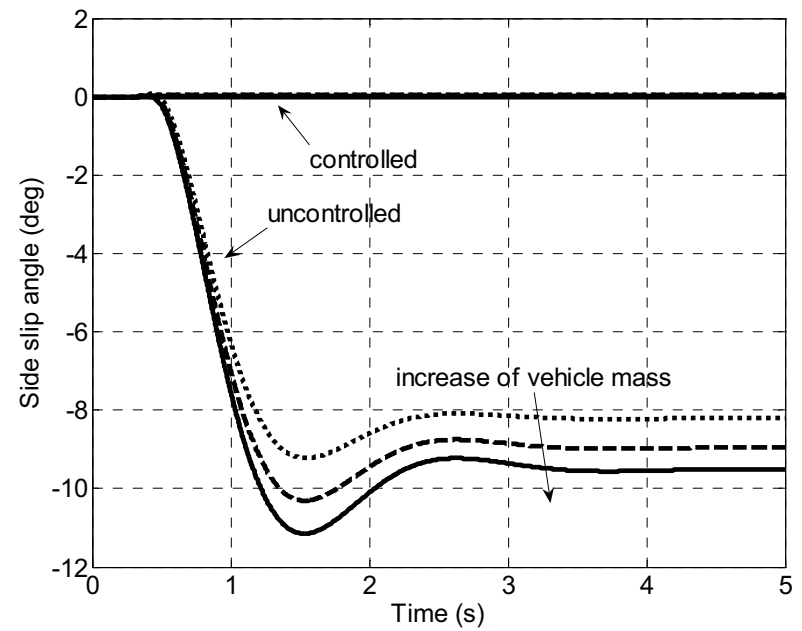

Figure 7: Simulation results for sideslip angle under J-turn manoeuvre

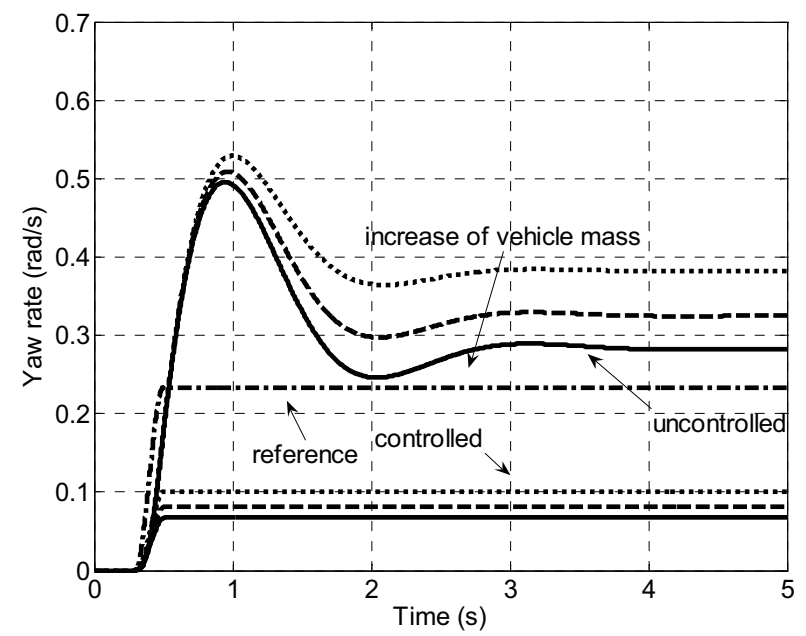

Figure 8: Simulation results for yaw rate under J-turn manoeuvre 


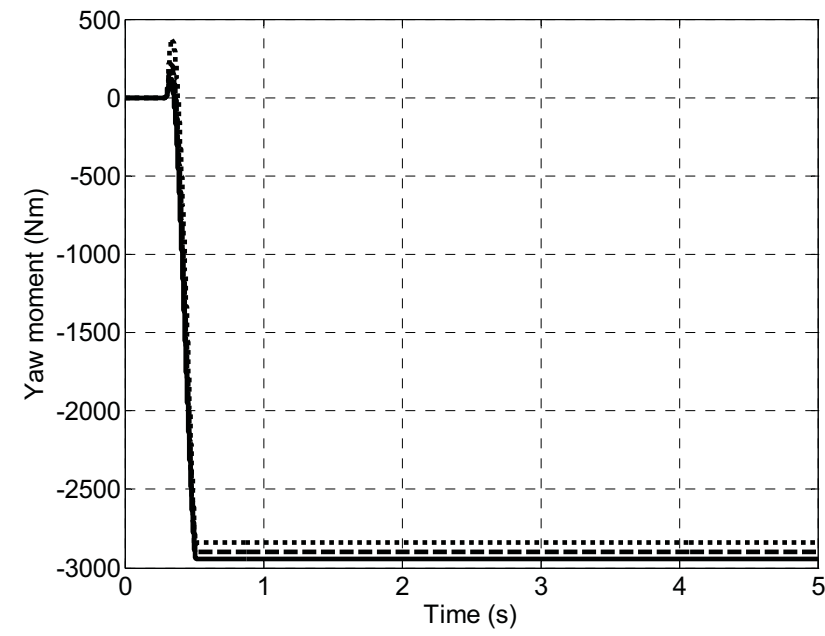

Figure 9: Simulation results for yaw moment under J-turn manoeuvre

\section{CONCLUSIONS}

This paper presents a robust controller design approach for vehicle yaw moment control considering vehicle load variation and control input constraint. Based on a 2DOF linear vehicle lateral dynamics model, the T-S fuzzy model is first applied to represent the vehicle load uncertainty. Then, a T-S fuzzy controller is designed under the constraint of control input. Considering the vehicle load variation and control input constraint in the controller design process provides a more practical realisation to improve the vehicle handling and stability. By solving the linear matrix inequalities, the required state feedback controller can be obtained very efficiently. Numerical simulations are used to demonstrate the effectiveness of the designed controller. Further study on this topic will be extended to the nonlinear vehicle dynamics model and consider more parameter uncertainties, such as cornering stiffnesses, moment of inertia, forward velocity, etc. More advanced method such as tensor product (TP) model transformation method will be applied to represent the nonlinear uncertain model. And then, robust controller will be designed to enhance vehicle handling and stability. On the other hand, it has been found in the simulations that a trade-off has to be made between sideslip angle and yaw rate in the presented study. How to make the two variables tracking their desired values simultaneously will also be studied in the next step.

\section{ACKNOWLEDGMENTS}

The financial support of this work by the Australian Research Council (DP0560077) is gratefully acknowledged.

\section{REFERENCES}

1. E. Esmailzadeh, A. Goodarzi, and G. R. Vossoughi (2003). Optimal yaw moment control law for improving vehicle handling. Mechatronics, 13:659675.

2. M. Eslamian, G. Alizadeh, and M. Mirzael (2007). Optimization-based non-linear yaw moment control law for stabilising vehicle lateral dynamics. Proc. Instn. Mech. Engrs. Part D, 221:1513-1523.

3. B. L. Boada, M. J. L. Boada, and V. Díaz (2005). Fuzzy-logic applied to yaw moment control for vehicle stability. Vehicle System Dynamics, 43:753770.

4. J. H. Park, and W. S. Ahn (1999). $\mathrm{H}_{\infty}$ yaw-moment control with brakes for improving driving performance and stability. Proceedings of the 1999 IEEE/ASME International Conference on Advanced Intelligent Mechatronics, 747-752.

5. M. Canale, L. Fagiano, M. Milanese, and P. Borodani (2007). Robust vehicle yaw control using an active differential and IMC techniques. Control Engineering Practice, 15:923-941.

6. H. M. Lv, N. Chen, and P. Li (2004). Multi-objective $\mathrm{H}_{\infty}$ optimal control for four-wheel steering vehicle based on yaw rate tracking. Proc. Instn. Mech. Engrs. Part D, 218:1117-1123.

7. S. S. You, and Y. H. Chai (1999). Multi-objective control synthesis: an application to 4WS passenger vehicles. Mechatronics, 9:363-390.

8. S. Zhang, H. Tang, Z. Han, and Y. Zhang (2006). Controller design for vehicle stability enhancement. Control Engineering Practice, 14: 1413-1412.

9. K. Tanaka and H. O. Wang (2001). Fuzzy Control Systems Design and Analysis. John wiley \& Sons, Inc.

\section{CONTACT}

Haiping Du

Mechatronics and Intelligent Systems, Faculty of Engineering, University of Technology, Sydney, P.O. Box 123, Broadway, NSW 2007, Australia. E-mail: hdu@eng.uts.edu.au 